\title{
LEARNING THROUGH VIRTUAL TOOLS: VISIT A PLACE IN THE PANDEMIC ERA
}

\author{
F. Fiorillo ${ }^{1}$, G. Rizzi ${ }^{1}$, C. Achille ${ }^{1 *}$ \\ ${ }^{1}$ Department of Architecture, Built Environment and Construction engineering, Politecnico di Milano, via Giuseppe Ponzio 31, \\ 20133 Milan, Italy - fausta.fiorillo, cristiana.achille@polimi.it, giulia1.rizzi@mail.polimi.it
}

KEY WORDS: Cultural Heritage, Virtual Tour, Remote Fruition, Remote Education, Distant Learning, Spherical Image, Immersive Photography, School-Work project

\begin{abstract}
:
The concept of combining learning activities and Cultural Heritage is a highly effective approach, both in the pandemic era and no. Knowledge and awareness of a place cultural identity by the local community is itself a conservation action. Therefore, a training activity was tested at the "Liceo Leopardi" (Lecco) according to the guidelines of Italian work-school projects. It is an innovative didactic methodology that allows students to combine school training with practical experience in a public or private institution. Since 2015, it has been compulsory in Italy for all high school students. The didactic tool used to face innovative topics related to heritage digitisation and its remote access was the Virtual Tour of the Monumental Complex of Laorca. The VT is a very effective immersive tool and easy to use even for non-expert users. Through some frontal lessons and laboratory activities, the students learned the basic concepts about the acquisition techniques to realise multimedia data (artistic photography, light painting, panoramic photography, video, etc.) to enrich the Laorca complex VT frame. The didactic experimentation was very engaging for the students who created a cultural game for the younger middle school students. The game is structured like a path consisting of 10 phases, designed in two versions: one version played directly on-site and another available online within VT. The research presented aims at the $\mathrm{CH}$ digitisation for the involvement and participation of the local community in the territorial identity promotion. The didactic experiment involved students reinforcing the link with their territory.
\end{abstract}

\section{INTRODUCTION}

The Cultural Heritage $(\mathrm{CH})$ and its safeguard and preservation are just a few priorities of modern society, "the safeguard consists in the exercise of functions and in the regulation of activities directed, based on an adequate cognitive activity, to identify the assets constituting the cultural heritage and to ensure the protection and preservation for purposes of public access" (D.L n.4, 2004). Public access, considered an integral part of $\mathrm{CH}$ safeguard, is one of the aspects suddenly denied due to the pandemic emergency. The latter has upset the regular daily routine and the usual social dynamics but has created new equilibrium, both professional and personal.

The $\mathrm{CH}$ sphere is undoubtedly one of the areas that have suffered the most since it is no longer possible to reach places of interest physically. There have been numerous initiatives of remote fruition of the heritages promised by national and international institutions and museums to overcome this social and cultural block and escape from the 'static' reality of the last year.

The research starts from a critical analysis of various solutions and initiatives of virtual exploration of cultural and natural places undertook by museums, institutions and communities. In particular, potentialities and possibilities provided by Virtual Reality (VR) current applications that ensure an immersive visiting experience were analysed (De Fino et al., 2020; Kersten et al., 2018; Napolitano et al., 218; Paladini et al., 2019).

The research interest is developed to consider these "cultural solutions' not temporary and contextualised to the current pandemic emergency but employable on a large scale as a powerful tool to support the enhancement and promotion of cultural identities and learning activities. The virtual environment is also often used to promote and make available generally unexplored places such as underwater heritage (Bruno et al., 2019).

Indeed the knowledge and the awareness of a place cultural identity by the local community is itself a preservation action. Therefore, the match between heritage safeguard and learning activities turns out to be effective, both during the pandemic era and not.

\section{CULTURAL HERITAGE AND EDUCATION: LEARNING HIGH SCHOOL PROJECT}

The idea of combining training and $\mathrm{CH}$ attracted the interest of high schools immediately, and it was decided to structure the training activity according to the guidelines of the Italian school-work projects.

The school-work is a teaching methodology that allows students to combine school training with practical experience in a public or private institution. Since 2015 in Italy, it has been compulsory (Legge n.107, 2015) for the students of high schools.

The school-work innovative method helps to consolidate the knowledge acquired at school and to test student aptitudes in the field through practical experience. These types of project activities enrich the student training and guide their study path and work in the future, thanks to projects in line with their curriculum. From 2019, the complete name of school-work projects is "Pathways for Transversal Skills and Orientation (PCTO - Percorsi per Competenze Trasversali e Orientamento)" (Alternanza, 2017).

\footnotetext{
${ }^{*}$ Corresponding author
} 
Based on specific agreements stipulated with companies, high schools and training institutes are required to organise for their students periods of professional training in the company or other activities that favour integration with the world of work (orientation days, meetings with companies and professionals, internships, field research, project work, etc.).

The school-work alternation is based on some well-defined objectives:

- to complement and integrate school training with the acquisition of more practical skills, in such a way as to favour a practical approach to the labour market;

- to help the orientation of young people enhancing their aspirations and interests;

- to help students develop the ability to choose autonomously and consciously;

- to support the student personal growth through extracurricular experiences that contribute to the development of a sense of responsibility.

A didactic tutor is appointed to ensure continuity between the training activities carried out at school and carried out not a school. Generally, it is a teacher who assists the students and verifies the correct development of the path in alternation between school and work. A tutoring company is also appointed, favouring the student insertion in the company and collaborates with the school allowing the activities verification. The learning project proposed and developed in the second half of 2021 meets universities active involvement, high schools and third sector associations. The training proposal focused on data acquisition techniques for creating multimedia content (images, video, audio, etc.) which enriched the already realised Virtual Tour (Rizzi, 2021) of the monumental complex of Laorca in Lecco.

The students carried out applied activities in the field and academic activities in the classroom. After a first necessary inspection to discover the area of interest (with a virtual and physical site exploration), a series of classroom lessons were developed (photography, image editing, illusions, light painting, panoramic images) to identify the better way to acquire the information to enrich the tour. The data collected in two subsequent field trips were also used to design a game path in stages proposed to the middle-class students.

\section{CULTURAL HERITAGE AND EDUCATION: LEARNING THROUGH VIRTUAL TOOLS}

\subsection{Background}

Different $\mathrm{CH}$ remote fruition modes, implemented by national and international museums and cultural institutions, were examined. Many virtualisation actions presented were implemented especially following the pandemic event.

The critical overview of virtual experience solutions and their strengths and weaknesses focused on the following main categories: i) VT hosted by "Google Arts \& Culture" (e.g. the London British Museum ${ }^{1}$ ) ii) customised VT of international museums (e.g. the Paris Louvre ${ }^{2}$ ) and cultural institutions (e.g. the Yosemite National Park in California ${ }^{3}$ ); iii) $360^{\circ}$ virtual video tours (e.g. New York Metropolitan Museum ${ }^{4}$ ); iv) animated $\mathrm{CH}$ video-presentation (e.g. Ferrara Palazzo Schifanoia ${ }^{5}$; v) online portal for browsing 3D models,

\footnotetext{
${ }^{1} \mathrm{https}: / /$ www.britishmuseum.org/collection/galleries

${ }^{2} \mathrm{https}: / /$ www.louvre.fr/en/online-tours

${ }^{3}$ https://www.virtualyosemite.org/

${ }^{4} \mathrm{https}: / /$ www.metmuseum.org/art/online-features/met-360-project

${ }^{5}$ https://www.youtube.com/watch?v=1fJ8DjDjDuU
}

integrated to the VT experience (e.g. Direzione Regionale Musei $\mathrm{Abruzzo}^{6}$ ); vi) and finally VT of 3D museum environment, not existing but created for the virtual visit experience (e.g. "Raphaello VR" Skylab Studios").

"Google Arts \& Culture" 8 is a non-profit initiative that, through collaboration with cultural institutions and artists worldwide, is aimed to preserve and bring art and culture online and accessible to anyone and anywhere. The project has many famous and prestigious partners, such as the Musée d'Orsay (Paris), The Metropolitan Museum of Art (New York), the Van Gogh Museum (Amsterdam), and many others equally important. This VT has the same fruition mode as Google Street View: it allows one to explore a $360^{\circ}$ scene and move from a panorama to another. There is no interaction during the scene exploration: no hotspots link to descriptive texts or other kinds of information, no map that can orientate the visitor.

The second category, on the other hand, refers to more open and customisable VT solutions. In the virtual visit of some museum rooms and galleries of the Louvre museum, some icons link to informative text and icons that link to detailed photos of some artworks. In addition, the visit is accompanied by introductive information (generally a descriptive presentation of the place and the web exploration mode) and a site map of the panoramic stations to guide the users during the navigation.

The third category regards the virtualisation of the visit through a $360^{\circ}$ video that imposes a pre-established path on the user. The fruition is made less rigid by the moving possibility at $360^{\circ}$ within the environment presented by the video, leaving yet to look around at along the fixed path.

On the other hand, animated video presentations are a very effective and attractive didactic tool to convey cultural information. In the example of Palazzo Schifanoia in Ferrara, the historical/architectural evolution of the building is narrated and represented. Nevertheless, this modality does not foresee any interaction with the user, who is therefore passive.

The fifth category concerns web portals that allow the visualisation of and direct interaction with $3 \mathrm{D}$ models, such as Sketchfab $^{9}$ and 3DHOP (3D Heritage Online Presenter) ${ }^{10}$. The 3D assets web presentation can be enriched with icons to add further information and descriptive texts.

In closing, "Raphaello VR" is a project by Skylab Studios (a visual communication studio) created at the beginning of the quarantine due to the Coronavirus. It is a virtual museum environment reconstructed in 3D to house 22 of the Maestro's main works.

The paintings visible on the walls of the museum are enriched with six different functions: textual information on the artwork to be read; Raphael's narrating voice that illustrates the painting history and details; lessons by a well-known art critic; the talking version of the painting created using morphing techniques where the portrayed protagonists come to life and tell their stories; the kid-friendly version, i.e. the cartoon version of the paintings that speak to children in a more simple language; and finally the video guide for the deaf in sign language.

The interactive space of the museum is free and open to everyone, 24 hours a day. It presents an interactive solution to involve people in the history of art and the life of Raphael and is an ideal didactic tool too.

\footnotetext{
${ }^{6}$ https://sketchfab.com/PoloMusealedellAbruzzo

${ }^{7} \mathrm{https}: / /$ www.skylabstudios.it/raphaellovr-raffaello-realta-virtuale

${ }^{8} \mathrm{https}$ ://artsandculture.google.com/

${ }^{9} \mathrm{https} / / /$ sketchfab.com/

${ }^{10} \mathrm{https}$ ://www.3dhop.net/contacts.php
} 


\subsection{Research aims}

The research presented aimed to digitise heritage for the local community involvement and participation in the territorial identity promotion. The question focuses on finding the most effective digital tool to bring cultural interest back to life and involve the local community in this virtual experience. This last aspect calls for a dedicated project/intervention that creates 'community links' with its place.

From this perspective, the Virtual Tour is an instrument of great immersive effectiveness and simple to use even for non-expert users. Therefore, the customisable type (like those of the second category) can be an efficient tool (and even a database) to share and visualise cultural/historical data and technical analysis too, e.g. actual state of conservation, state of maintenance, decays information, etc. (Cantatore et al., 2020). In our case, the VT revolutionary aspect is that the heritage approaches people by entering their homes and not vice versa, as it has always been until now.

Local didactic activities (mixed, remote and in presence) have been structured using the VT as a teaching experiment to achieve this primary aim. The topics developed concern the heritage digitalisation techniques applied to a local cultural asset that has to be valorised.

Both the animated video presentation and the 3D models in the web page (categories iv and $\mathrm{v}$ of the previous paragraph) are two ways of remote access that can be integrated within the structure of a VT. In fact, during the teaching activities, both were illustrated and proposed as ideas to enrich the basic frame of the chosen case study tour. On the other hand, the last example was especially useful in raising awareness of a simplified approach for kids or users with disabilities.

The proposed examples demonstrate the growing interest in the possibilities of $\mathrm{CH}$ remote access and are excellent models of innovative applications at the service of culture.

\section{CASE STUDY}

\subsection{The Monumental Complex of Laorca}

The Monumental Complex of Laorca in Lecco (Rota, 2014; Bonfanti, 1990) is the case study chosen to realise a VT of the visit route, much loved by the local community. Laorca is the northernmost neighborhood of Lecco. The area in which the complex rises is of strong suggestion (Figure 1): it is located at the base of the Corna Medale, rocky spurs in which caves and ravines open, creating a sort of natural amphitheatre that hosts the cemetery, the "San Giovanni ai Morti" church, the "Madonna di Lourdes" chapel (carved into the rock) and a panoramic terrace. The Via Crucis, formed by 14 chapels/stations, develops along the slope towards the cemetery up to the church. Moreover, the monumental cemetery complex, a unique historical-environmental heritage, has been included in the European Route of Significant Cemeteries.

\subsection{The Virtual Tour}

In this context, the need is twofold: to digitise and explore nature and architecture. In this direction, panoramic photography (Figure 2) ensures an efficient immersion in the landscape, both natural and monumental, offering an intuitive and accessible $360^{\circ}$ exploration even at different scales. Besides, the VT ensure moving among the spherical views to browse the site. At the same time, the VT structure allows the integration in the panorama of different types of data (textual information, 2D images, video animations, and even 3D models), creating an information-rich interactive scene (Walmsley and Kersten, 2020).

The equipment generally used to construct spherical images consists of: i) reflex camera and fisheye lens whose wide Filed Of View allows to reduce the number of shots needed to recompose the $360^{\circ}$ view; ii) panoramic head to remove parallax errors (by adjusting the coincidence between the optical center and the nodal point).

The multiple single shots are then combined and merged to produce a panorama in a spherical or equirectangular projection (a view of $360^{\circ}$ horizontally and $180^{\circ}$ vertically, i.e. of the entire sphere). When a spherical image is projected onto a plane, the horizontal lines appear curved while the vertical lines remain straight (Figure 2). The photographs are matched and aligned according to a homography by identifying homologous points between single photos (Brown and Lowe, 2007). For a seamless panorama, the various parts must be cut regarding common edges and have the same exposure (digital blending).

Two types of equipment were experimented with to create the monumental complex of Laorca VT ${ }^{11}$ (Figure 3): the $360^{\circ}$ panoramic camera NCTech's iSTAR pulsar and the action cam GoPro HERO7 Black.
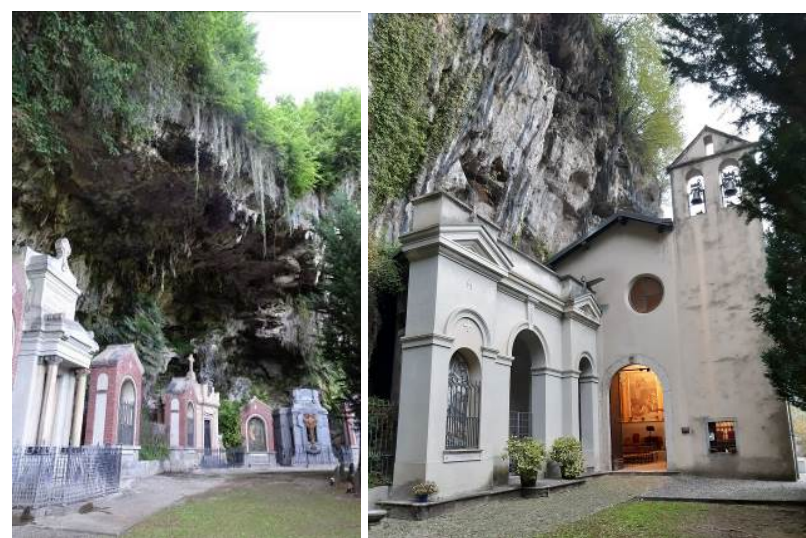

Figure 1. The Laorca monumental complex (the Via Crucis on the left, and the "San Giovanni ai Morti" church on the right).

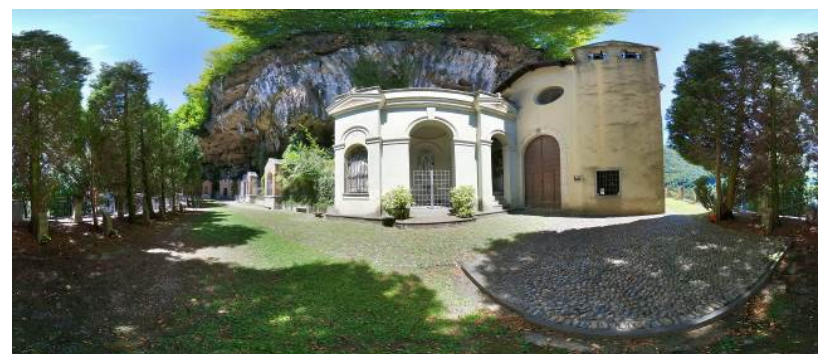

Figure 2. Equirectangular image of a point view near the "San Giovanni ai Morti" church.

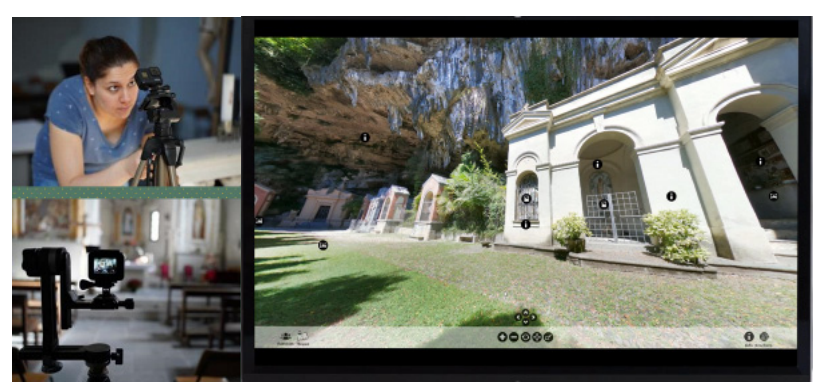

Figure 3. On site acquisitions and VT navigation.

${ }^{11} \mathrm{https}$ //giuliarizzi.altervista.org/laorca/ 


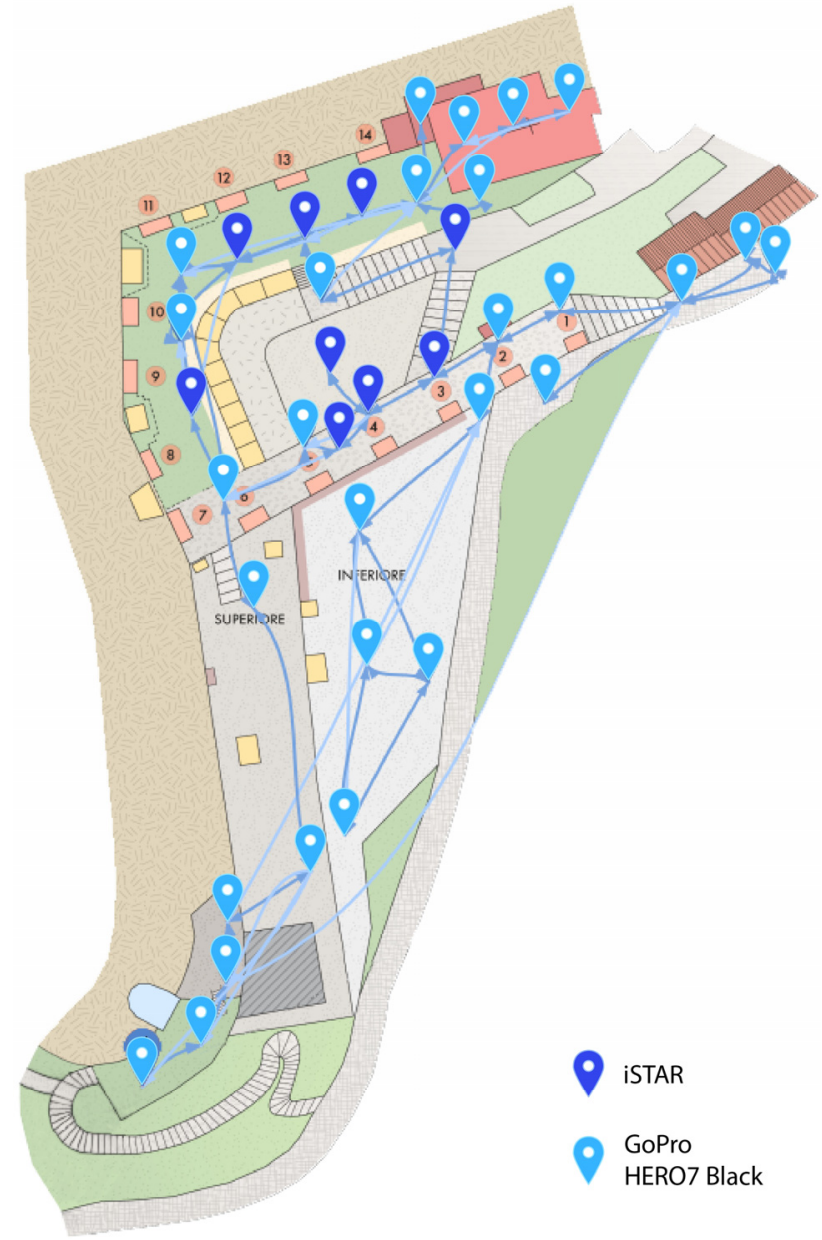

Figure 4. Laorca VT site map with the Via Crucis chapels, the panorama point stations and the links among the spherical views.
The tour consists of 37 spherical panoramas shows on the site map of Figure 4. Ten panoramas were made with the iSTAR and symbolised with dark blue icons. The remaining 27 made with GoPro HERO7 Black are represented in light blue.

The anodised aluminium iSTAR camera is compact, light (a cube of $10 \mathrm{~cm}$ side by side for a weight of $1.41 \mathrm{Kg}$ ), easily transportable and positionable. In addition, the simultaneous and independent capture by four fisheye lenses covering a Field Of View (FOV) of $360^{\circ} \times 275^{\circ}$ ensures a remarkable operating speed (the shooting time is 8 seconds per single exposure).

The camera setting allows the management and customisation of a very few parameters (such as the ISO and different exposure modes: manual, indoor, outdoors, front outdoors). The focus and the aperture are fixed. The High Dynamic Range (HDR) function allows five bracketing exposures (the max and min exposure time range is automatic).

The final panoramic image has a resolution of 50 megapixels, approx. $10,000 \times 5,000$ pixels. The image processing (stitching and blending) for the shot merging and panorama production is automatic with the house software (NCTech Immersive Studio). Although the instrument operating speed, ease of use, handling and flexibility are extraordinary, in outdoor captures with high contrast between shadowy areas and high lights, it was challenging to obtain panoramas with good exposure, despite the automatic HDR. In addition, the GoPro spherical images result in a slightly higher resolution and a better exposure condition. Figure 5 shows a comparison between two panoramas where it is also evident that the HDR iSTAR panoramas have a less natural colour (attractive like artist effect but not realistic). Therefore, only 10 iSTAR panoramas were used for the VT, which helped make the visit richer and more comprehensive anyway.

For this research activity, instead of an SLR camera and fisheye, the GoPro is used, whose wide FOV ensures similar performances, allowing the creation of a spherical image with a limited and manageable number of shots. The action cam is also much smaller and lighter, making it easier to handle, especially outdoors, where shooting conditions are not always easy.
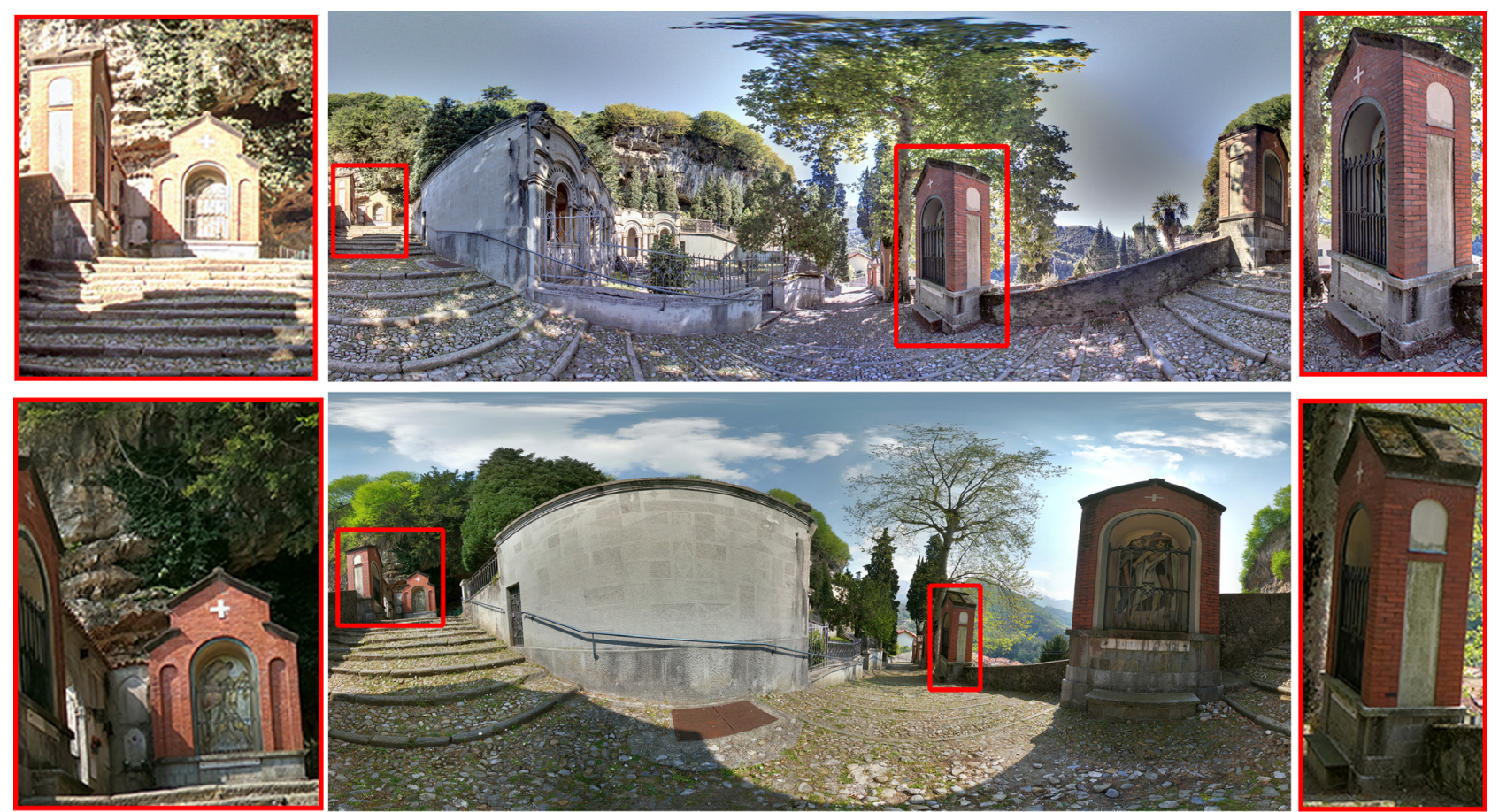

Figure 5. Comparison between the iSTAR (top) and GoPro panoramas (bottom). 
The camera ease of handling, low cost and tight deadlines were the three factors that led to the use of the GoPro. Moreover, it also allows for more impressive and suggestive views positioning the camera overhang. In fact, for the pandemic emergency, it was necessary to create the frame of the VT as soon as possible to start the remote learning experiences.

The GoPro HERO7 has a $94.4^{\circ} \times 122.6^{\circ}$ FOV (focal length of $2.92 \mathrm{~mm}$, a crop factor of 5.62 and equivalent focal length of $16.4 \mathrm{~mm}$ ). The number of photographs taken to compose a panorama was 15: 6 horizontal shots with a range of $60^{\circ} ; 4$ tilted of $+35^{\circ}$ and 4 tilted of $-35^{\circ}$ with a range of $90^{\circ}$; 1 zenith shot to be sure to close the panorama.

The GoPro photos stitching (homologous points automatic recognition and match) and the related digital blending (singleshot exposure compensation for a more uniform exposed panorama) was realised with the software Kolor Autopano Giga (v4.4.2). Instead, the VT was designed using the Pano2VR (v6.1.10). Figure 4 also shows the deliberately redundant links created among the different stations and designed for free navigation/exploring within the virtual environment.

The detailed photographs within the tour were taken with the Sony Alpha 7RIV camera and 28-75 mm lens. Indeed, the VT is enriched by additional photos, documentation and textual information thanks to specific hotspots within the single spherical images. Each extra element inserted in the panorama has different icons, which characterises it and differentiates it from the other information displayed.

\section{REMOTE ACCESS AND LEARNING}

The structure of the VT created was used as a didactic tool to start a project of excellence at the "Liceo Leopardi" (high school) in Lecco (in the Laorca district) on the issues related to the web tour and the related multimedia contents. During the quarantine, not to suspend the didactic activities, the on-site visit was replaced by the VT web navigation (Figure 6). This way, the students were also able to familiarise themselves with the instrument. Then they used the collected data, both multimedia and non-media, to design a game path in stages proposed to the middle school students.

Through some frontal lessons and on-site laboratory activities (Figure 7), the students learned the basic concepts about the data acquisition techniques to realise multimedia contents (artistic photography and light painting, panoramic photography, video, etc.) that enrich the Laorca complex VT.

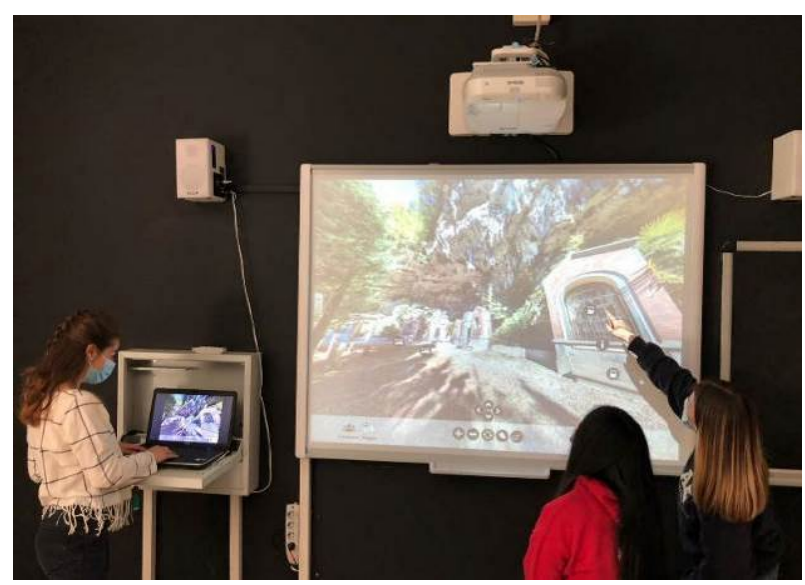

Figure 6. Exploring the Laorca Complex: remote class trip.

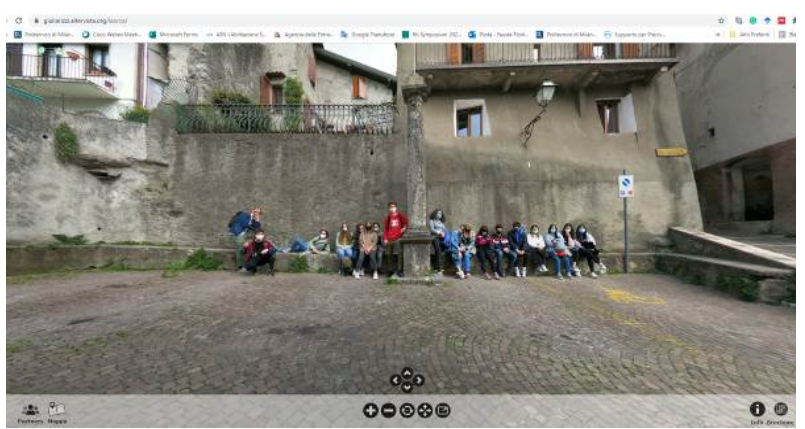

Figure 7. On-site activities during the student laboratory.

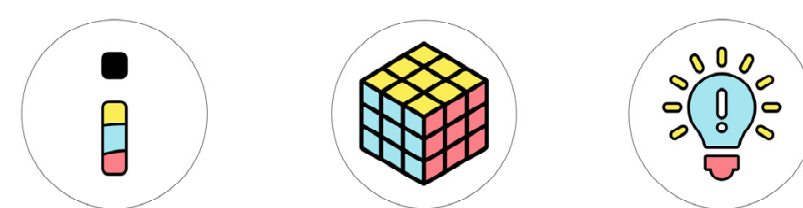

Figure 8. Game icons: from left to right, the additional historical/cultural information related to the single-stage, the link to the game and the solution.

The didactic experimentation was very engaging for the students, who managed to create a path consisting of 10 game stages, designed in two versions: one version to be played directly on-site; and another available online within VT.

Each stage of the game is related to a topic, legends and historical/architectural/natural details that intrigued the students during the visits in person and remotely.

The chosen subjects were then studied and deepened by the students (Bonfanti, 1990) and verify with their professors.

For the online version of the entertainment, three icon types (Figure 8) have been added at the panoramic station where the game stage has to take. The first type of icon is for the additional historical/cultural information related to the singlestage; the second to link to the game; the third for the solution. This last icon appears in the panorama, however, only after closing the game window.

The game first stage (the column of stubborn ones) is positioned in the forecourt of "Santi Pietro e Paolo" church before the Via Crucis start. The local anecdote narrates that all Laorca inhabitants are stubborn because, after the baptism, their heads are 'knocked' on this famous column in front of the church. The story is told through a cartoon bubble hand-drawn and presented randomly. The game aims to reorder the comic strip boxes to recreate the story (Figure 9).

The second stage is a word search puzzle associated with the Via Crucis starting point. The keywords refer to a descriptive text of the chapels.

The third stage is associated with chapels 6 and 7 (Figure 4). Their stylised photos are compared to find the differences.

The fourth stage occurs in front of the second chapel (Figure 4), near a memorial to fallen for the homeland. The idea is to bring to life the cartoon of a soldier telling the meaning of a memorial. The game is a quiz with multiple-choice questions about the explanation presented by the soldier. The fifth stage takes place along Viale delle Rimembranze.

The students reproduced with the light painting technique some symbols that recur in the context (shell, cross, angel, the letters alpha ki omega). The game aims to find and associate the icons light drawn in the VT environment. The sixth stage takes place along the last stretch of the avenue between chapels 11 and 14 (Figure 4). The game aims to match the stylised symbols to the corresponding textual definitions. 


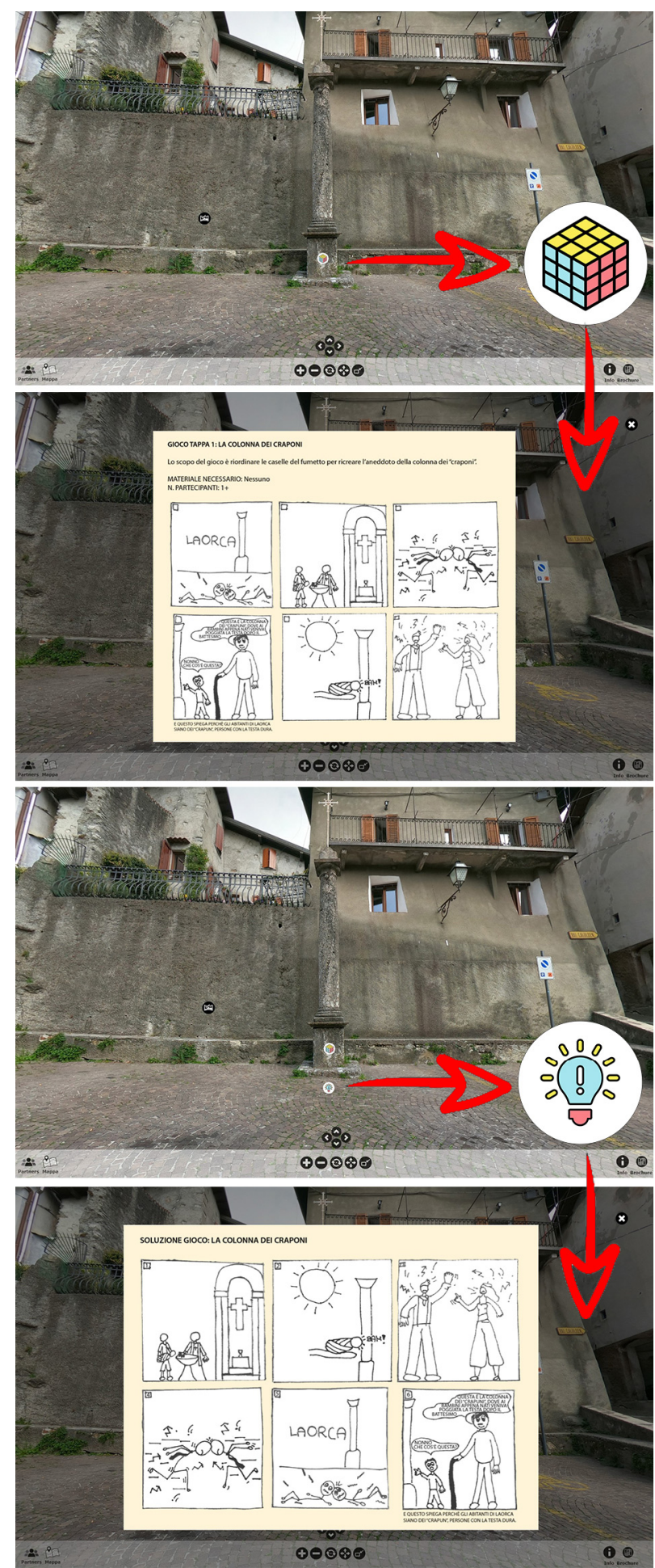

Figure 9. The game first stage (the column of stubborn ones)

The seventh step is at the 'miraculous chalice' in the churchyard of San Giovanni. The inhabitants of Laorca believe that the water collected in the chalice is miraculous and helps to preserve sight. To link up with visual skills, the students created shadows of some in-site symbols and other children subjects. The game aims to guess to whom the shadows belong. The eighth stage takes place in the last chapel between the church and the Ossuary. The chapel is also known as the Scurolo, which was the place where the venerated hermit John took refuge. The game aims to complete with the missing words a text that retraces the legend of the hermit.

The ninth stage, called "The Metamorphosis", takes place inside the church near the statue of Job. According to legend, God, to repay Job's faithfulness, transformed the worms of his plagues into silkworms (Figure 10). The game aims to order the pictures representing the life cycle of a caterpillar that becomes a butterfly.

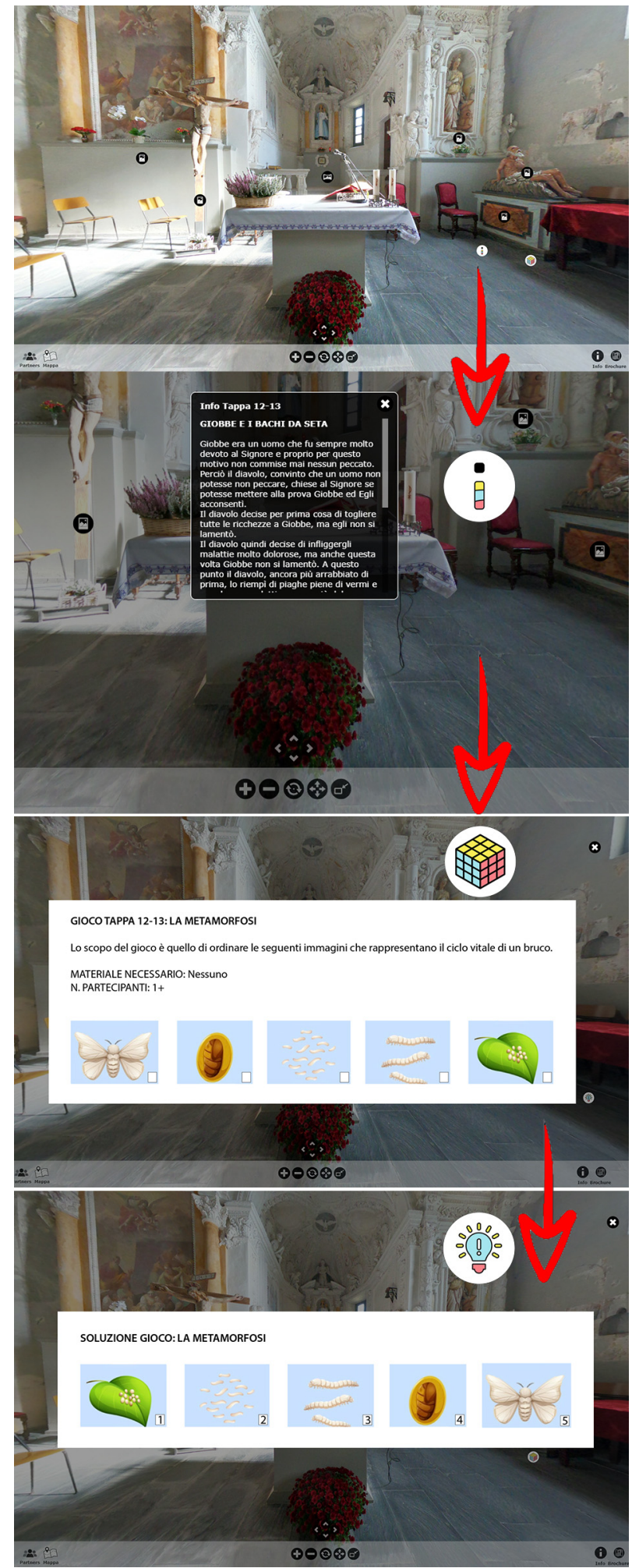

Figure 10. The ninth stage: the game of metamorphoses. 


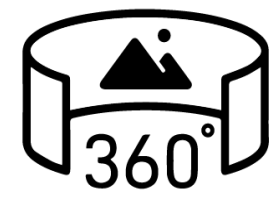

\section{Virtual Tour}

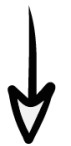

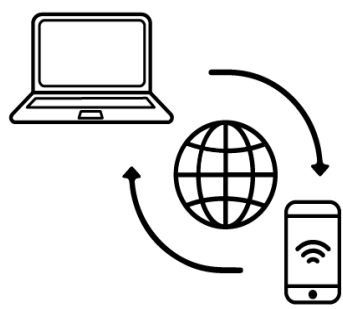

Dissemination and Remote Access

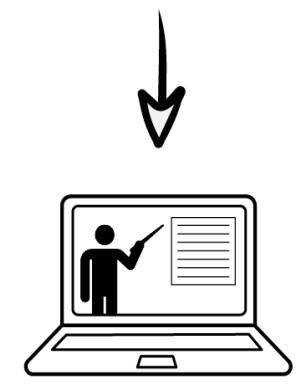

\section{Didactic Actions for the local community on the territory}

Figure 11. Functional diagram of an 'alive' VT application: lived, enjoyed, updated and enriched by the local community.

The last step is on the panoramic terrace, where the players have to complete a crossword puzzle while enjoying the view of the city of Lecco. The questions refer to the city and the monumental complex remotely explored.

The description of the game stages devised by the students was presented to illustrate the response of the students to a new didactic stimulus.

The creativity and flexibility with which they have proposed games connected to the site and playable online are highlighted. Furthermore, the designed games involved many personal skills and very different interests, ranging from a freehand drawing of balloons to light painting techniques.

The game path was an additional and very successful initiative to involve and bring the community of all ages closer to discovering the Laorca complex.

\section{CONCLUSIONS}

In the months of lockdown, with the suspension of teaching, the school world has been catapulted into a new dimension. With its means and limits, the digital has taken the field to help the educating community face the emergency.

The pandemic emergency has increased and pushed virtual approaches and technologies to disseminate and promote historical, archaeological, and natural sites.

The lockdown made us feel the lack and at the same time rediscover the pleasure of a cultural visit, even if virtual.

Through VR, it has been possible to make all those cultural places that are physically inaccessible at the moment accessible to anyone. The monumental complex of Laorca represents the identity of the local community; documenting and making the site digitally accessible has made it possible to keep the contact between the community and its heritage alive and constant. The project does not want to limit itself to the mere technological experiment of creating a VT but wants to be the starting point for new didactic projects that see the central role of $\mathrm{CH}$ and central use of technologies that, in addition to documenting the state of conservation, can contribute to their enhancement.

The tour was used to replace the class trip not possible with a 'virtual trip', to make the site known and stimulate the creativity of the students to produce multimedia content that enrichs the virtual visit.

Figure 11 shows the functional diagram of a not "static" but dynamic/alive VT, which is not just a technological application and may soon be not used and forgotten. Instead, a virtual space lived, enjoyed, updated and enriched by the local community.

The organisation of the blended PCTO course allowed students to test what they learned in the classroom through a concrete experience in both a real and simulated context.

At the end of the activities, each student gave his personal account of the experience, highlighting their point of view and focusing attention on some aspects: what was positive and what was negative? Have I achieved the goals set? What would I like to be improved and how? What are the critical issues, and what are the solutions?

Active dialogue with teachers and students is necessary to improve objectives and methods of delivering activities.

With the start of school in September, the school-work will also restart, probably still in a mixed form (presence-distance).

To continue the PCTO path effectively, it is necessary to carefully plan the activities, starting from the observations of the students at the end of this experience.

The emergency linked to the pandemic has made it possible to rediscover the importance of promoting 'proximity' projects for the rediscovery and protection of cultural heritage.

With these projects, the school encourages training to enhance $\mathrm{CH}$ observation, documentation and care skills.

The didactic experiment carried out up to now involved the students by strengthening the connection with their territory. The learning project has been structured to be implemented again in the future, both in the presence and remotely.

\section{ACKNOWLEDGEMENTS}

For the realisation of the project, an agreement was stipulated which involved: the Scientific Committee of the high school Liceo Leopardi in Lecco (headmaster Professor Paola Perossi); the Politecnico di Milano, Polo di Lecco (Professor Giuliana Iannaccone), the APS LaorcaLAB Association and the welfare services of the Municipality of Lecco.

The Authors thank all the students who have joined the project for their enthusiasm and passion. 


\section{REFERENCES}

Alternanza scuola-lavoro, 2017. Ministero dell'Istruzione e dell'Università della http://www.alternanza.miur.gov.it/cos-e-alternanza.html, http://www.alternanza.miur.gov.it/normativa.html (30 June 2021).

Bonfanti, A., 1990. A centonove passi dalla Chiesa di Laorca (fede e storia intorno alle grotte), Tipo-litografia Alfredo Colombo, Lecco.

Brown, M., Lowe, D.G., 2007. Automatic Panoramic Image Stitching using Invariant Features. Int J Comput Vision, 74, 5973. doi.org/10.1007/s11263-006-0002-3.

Bruno, F., Lagudi, A., Barbieri, L., Cozza, M., Cozza, A., Peluso, R., D., Petriaggi, B., Petriaggi, R., Rizvic, S., and Skarlatos, D., 2019. Virtual Tour in the Sunken "Villa con Ingresso a Protiro" Within the Underwater Archaeological Park of Baiae. Int. Arch. Photogramm. Remote Sens. Spatial Inf. Sci., XLII-2/W10, 45-51. doi.org/10.5194/isprs-archives-XLII-2W10-45-2019.

Cantatore, E., Lasorella, M., Fatiguso, F., 2020: Virtual Reality to Support Technical Knowledge in Cultural Heritage. The Case Study of Cryptoporticus in the Archaeological Site of Egnatia (Italy). Int. Arch. Photogramm. Remote Sens. Spatial Inf. Sci., XLIV-M-1-2020， 465-472. doi.org/10.5194/isprs-archivesXLIV-M-1-2020-465-2020.

De Fino, M., Ceppi, C., and Fatiguso, F., 2020. Virtual Tours and Informational Models for Improving Territorial Attractiveness and the Smart Management of Architectural Heritage: the 3D-Imp-Act Project. Int. Arch. Photogramm. Remote Sens. Spatial Inf. Sci., XLIV-M-1-2020, 473-480. doi.org/10.5194/isprs-archives-XLIV-M-1-2020-473-2020.

Decreto Legislativo 22 gennaio 2004, n. 42. Codice dei beni culturali e del paesaggio, ai sensi dell'articolo 10 della legge 6 luglio 2002, n. 137. G.U. n. 45, 24 February 2004, S.O. n. 28. https://www.camera.it/parlam/leggi/deleghe/testi/04042dl.htm (30 June 2021).

Legge 13 luglio 2015, n. 107. Riforma del sistema nazionale di istruzione e formazione e delega per il riordino delle disposizioni legislative vigenti. G.U. Serie Genrale n. 162. https://www.gazzettaufficiale.it/eli/id/2015/07/15/15G00122/sg (30 June 2021).

Kersten, T.P., Tschirschwitz, F., Deggim, S., Lindstaedt, M., 2018: Step into Virtual Reality - Visiting Past Monuments in Video Sequences and as Immersive Experience. In Latest Developments in Reality-Based 3D Surveying and Modelling, MDPI Books, pp. 192-218.

Napolitano, R.K., Scherer, G., Glisic, B., 2018. Virtual tours and informational modeling for conservation of cultural heritage sites. Journal of Cultural Heritage, 29, 123-129. doi.org/10.1016/j.culher.2017.08.007.

Paladini, A., Dhanda, A., Reina Ortiz, M., Weigert, A., Nofal, E., Min, A., Gyi, M., Su, S., Van Balen, K., Santana Quintero, M., 2019. Impact of Virtual Reality Experience on Accessibility of Cultural Heritage. Int. Arch. Photogramm. Remote Sens. Spatial Inf. Sci., XLII-2/W11, 929-936, https://doi.org/10.5194/isprs-archives-XLII-2-W11-929-2019.
Rizzi, G., 2021. Visitare un Bene Culturale a distanza. Tour virtuale del Complesso monumentale di Laorca (LC). Master's thesis, School of Architecture Urban Planning Construction Engineering, Politecnico di Milano. https://www.politesi.polimi.it/handle/10589/174313 (30 June 2021).

Rota, T., 2014: Percorsi di arte, storia e paesaggio nei cimiteri di Lecco. Amici dei Musei del territorio Lecchese, Lecco.

Walmsley, A, Kersten, T.P., 2020: The Imperial Cathedral in Königslutter (Germany) as an Immersive Experience in Virtual Reality with Integrated 360 - Panoramic Photography. Applied Sciences, 10, 1517. doi.org/10.3390/app10041517. 